\title{
Vulnerability Analysis of Satellites in Strong Electromagnetic Environment GUO Li-wen ${ }^{1 a}$, ZHANG Zhan-yue ${ }^{2}$, ZHANG Zhe ${ }^{1}$
}

1. Department of Graduate Management, Academy of Equipment, Beijing 101416,China; 2. Department of Space Command, Academy of Equipment, Beijing 101416, China a.email: guoliwen_exclusive@163.com

Keywords: Electromagnetic pulse (EMP), Satellites, front door coupling, back door coupling

\begin{abstract}
This paper, based on the damage effect of electromagnetic pulse (EMP) on satellites, firstly analyzes electromagnetic leakage elements of typical satellites, emphasizes the coupling process between EMP and satellites and its damage effect on satellites, and conducts preliminary simulation on antenna coupling in front door coupling and star sensor coupling in back door coupling, respectively. The simulation results show that when EMP power reaches certain strength in the strong electromagnetic environment, it will cause damage with varying degrees on satellites, which carry out no anti-electromagnetic reinforcement.
\end{abstract}

\section{Introduction}

EMP is a powerful energy field, which is generated instantly. It spreads in the form of space radiation, its frequency spectrum covers a wider range, and it can be generated in multiple forms, including thunder and lightning, high power microwave bombs, nuclear explosion and EMP bombs, and so on ${ }^{[1]}$. Because the front waveform of EMP is steep and has shorter risetime, high field intensity, wide range of action, it is a relatively stronger electromagnetic interference source. According to statistics of Satellite Industry Association, it can be found that nowadays, most of low earth orbit satellites in operation receive no anti-high power EMP reinforcement ${ }^{[2]}$. If they are disturbed or attacked by high power EMP, EMP will couple into the inner satellite system in any approach, so that satellite-bone electronic components will be disturbed or even damaged ${ }^{[3]}$.

\section{Electromagnetic Leakage Element Analysis of Typical Satellites}

Satellites generally are composed of satellite platform, transceiver antenna and solar panel, etc. The external structure diagram of a satellite is shown in Figure 1. From the perspective of system structure, a satellite is formed by a support system (which ensures normal operation of the satellite) and an effective load (which completes specific space tasks) ${ }^{[4]}$. If any key point is damaged, its influences on the entire working performance of the satellite may be immeasurable. It can be observed from the analysis of the satellite that potential entry positions of EMP are quite a lot, thus if a satellite carries with the control antenna, start sensor, optical imaging device, infrared camera , and digital antenna, etc., the coupling approach may be hard to be predicted. 


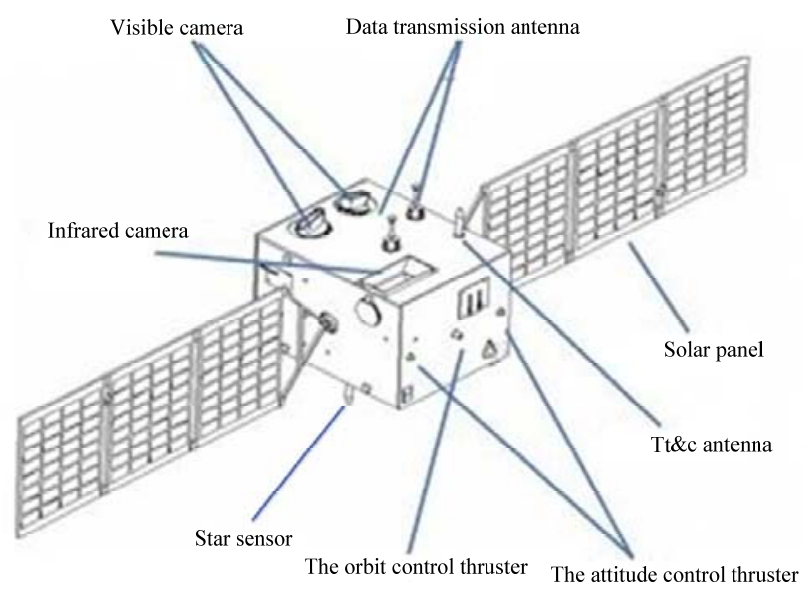

\subsection{Antenna Reception}

Figure 1: The Structure Diagram of Satellite Structure

For example, if the energy spectrum of EMP belongs to the scope of working band of antenna, energy of EMP will be sent to the inner receiver directly in the way of antenna coupling. The low noise amplifier that locates in front high frequency of a receiver is the easiest damaged part. If the part is damaged, it will cause disturbance or destroy to satellite communication system.

\subsection{Cables}

There are a great number of cables crossing the cabin out of the satellite. After EMP couples with it, EMP will enter into the inner satellite along the road. If the energy researches a certain degree, it will burn terminal cables. Moreover, cables with special configurations amount to the linear an antenna, so the radiant energy will disturb surrounding sensitive apparatuses.

\subsection{Optical Devices}

As a required optical device in the self-positioning of the satellite, star sensitive part of transparent window is used for receiving visible light, but also causes electromagnetic leakage. It may have higher degree of sensitivity and is extremely easier to be disturbed, saturated or even damaged by comparing with other electro-optical systems.

\section{4 Apertures}

At present, the cabin satellites, generally speaking, will adopt metal shell to electromagnetic interference to protect satellite control system in the cabin and communication instruction systems, etc., key electronic components. However, shield cabin generally has hole and seam with small size for cable connection or other purposes, while these apertures will cause electromagnetic leakage with varying degrees. These apertures will cause electromagnetic leakage with different degree, resulting in reducing the shielding performance of metal shielding layer. Therefore, shielding will reduce space effectively and make shielding effectiveness in metal shielding layer will be reduced.

\section{The Radiation Coupling Modes and Coupling Effects of the Satellites' Coupling Ways and Coupling effect.}

\subsection{The Coupling Path}

It can be observed from the elements of electromagnetic leakage that the main approach for EMP to enter into the inner satellite system, including antenna, optical components, cables and apertures. It can be divided into two ways, including front door coupling and back door coupling. The front door coupling means EMP energy enters into the inner satellites, while the back door coupling refers to the process that EMP energy enters into the inner satellites by virtue of antenna or the receiving system, EMP energy enters into the inner satellite. On the other hand, back door coupling refers to a process that apertures optical windows and various transmission lines, etc., substances, as well as various transmission lines will enter into the inner satellite ${ }^{[5]}$.

The coupling between EMP and the category is shown in Figure 2. First of all, high power EMP, antenna, cables, optical windows and various aperture couples enter into the effective load system 
of satellite bone. Thus, electromagnetic field forms the transient electric field in the inner cavity of the system, and coupled energy will conducting or radiating to inner sensitive fragile parts of electronic components to the satellite cabin by virtue of transition cable, from cables and waveguides. Ultimately, the fragile sensitive components in EMP and cabin will interact. If the power or energy of EMP is higher than the damaged threshold value of electronic components, electronic components or devices in the cabin will be disturbed, and working efficiency will be reduced. As seriously, it will damage electronic components, resulting in failing to work for the entire satellite system.

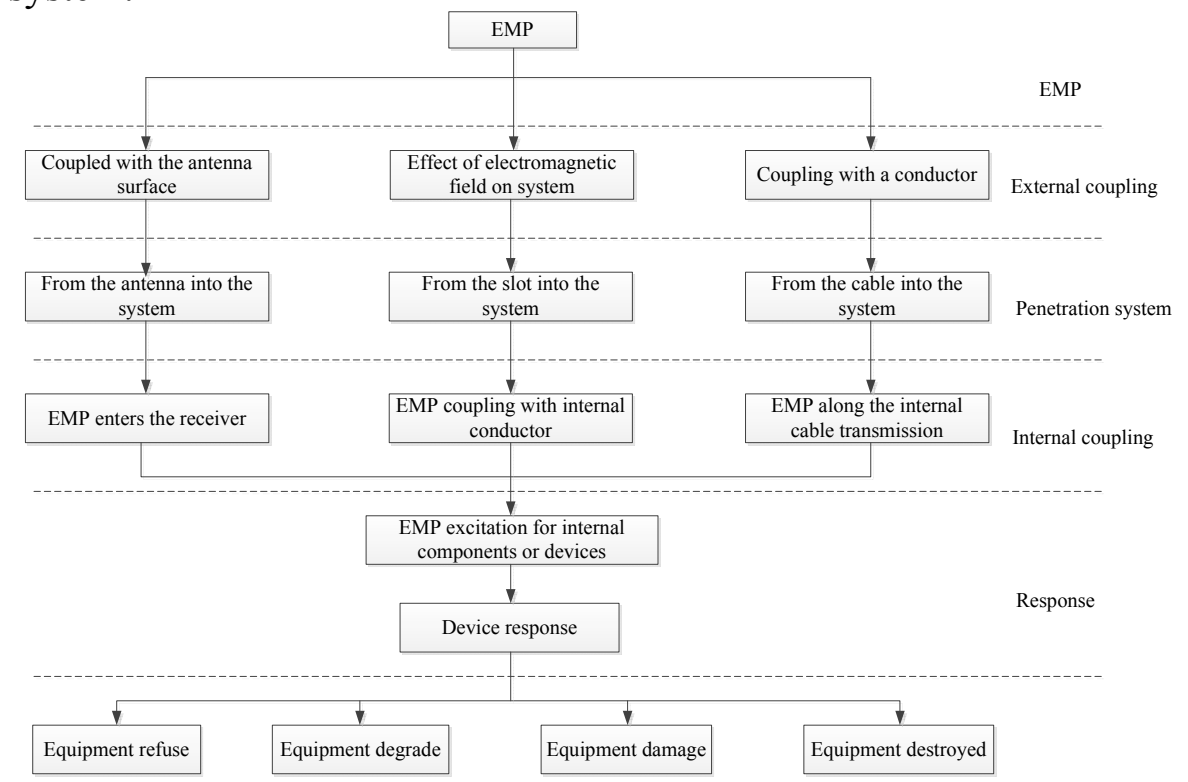

\subsection{Coupling Effect}

Figure 2: Approach and Process between EMP and Satellite Coupling

The influences of EMP on satellite system not only are related to power density of satellites, but also are relative to energy value of inner electronic components in satellite system and damaged threshold value of electronic components. Its influences on satellites will be divided into several categories in line with coupling and power of inner EMP in satellite system, as shown in Table $1^{[6]}$.

Table 1 The Influences of EMP on Satellites

\begin{tabular}{|c|c|c|c|}
\hline $\begin{array}{c}\text { Power } \\
\text { Density }\left(\mathrm{W} / \mathrm{cm}^{2}\right)\end{array}$ & Coupling path & Coupling effect & Coupling effects \\
\hline $0.01 \sim 1 \mu$ & $\begin{array}{c}\text { Enter from antenna } \\
\text { to disturbance }\end{array}$ & Disturbance & $\begin{array}{c}\text { Radar, communication devices, navigation systems } \\
\text { can't operate normally. }\end{array}$ \\
\hline $0.01 \sim 1$ & $\begin{array}{c}\text { Absorb microwave } \\
\text { energy to radiate }\end{array}$ & Radiation & $\begin{array}{c}\text { Detection, C4ISR and components and chips in arm } \\
\text { systems are failed. }\end{array}$ \\
\hline $10 \sim 100$ & $\begin{array}{c}\text { Induce microwave } \\
\text { current ionization }\end{array}$ & Ionization & $\begin{array}{c}\text { Transient electromagnetic field formed by } \\
\text { high-frequency microwave radiation will make metal } \\
\text { surface generate induced current and damage various } \\
\text { sensitive components. }\end{array}$ \\
\hline $10^{3} \sim 10^{4}$ & $\begin{array}{c}\text { Instant internal } \\
\text { combustion or break } \\
\text { out high } \\
\text { temperature }\end{array}$ & High temperature & $\begin{array}{c}\text { Strong electromagnetic field cause series of } \\
\text { non-linear effects, within short time, targets are } \\
\text { damaged by high temperature. }\end{array}$ \\
\hline
\end{tabular}

Thus, it can be observed that under the strong EMP, when EMP power density is different, its influences on satellites also are diverse. EMP is easy to disturb or damage satellites.

\section{Simulation Analysis of Satellites on EMP Coupling}

\subsection{Antenna Coupling}

For front door coupling, this paper is based on the EMP and antenna coupling. The energy receiving in the engine cavity of satellite is the parts of energy, when EMP covers working frequency of targeted satellites in antenna. The energy that is coupled to the satellite-bone system through antenna main lobe is shown as ${ }^{[7]}$ : 
In the formula, $\mathrm{P}_{\mathrm{r}}$ is the power coupling into the inner satellite-bone system. $\mathrm{P}_{\mathrm{e}}$ is the peak power of EMP, $B_{e}$ is the bandwidth of EMP, $G_{e}$ is the gain of EMP radiating antenna. $\lambda$ is the wave length of EMP, $G_{r}$ is the gain of satellite receiving antenna, $\mathrm{Br}$ is bandwidth of receiving antenna, and $\mathrm{L}$ is the transmission loss from antenna coupling to the cavity. In theory, L includes scattering loss in space(or space propagation loss) and feeder transmission loss, etc., given that EMP has a larger threat on satellites, so here only considers the space propagation loss of EMP, and the expression is shown as:

In the formula, $\mathrm{f}$ is the emission frequency of EMP weapons(unit: $\mathrm{MHz}$ ); $\mathrm{d}$ is the propagation distance of EMP(unit: $\mathrm{Km}$ ).

This formula can be regarded as the basic judgment formula for satellite-bone system whether suffers from damage of EMP, namely if electromagnetic energy conducted inner coupling in satellite-bone system is greater than damaged threshold value of front sensitive components of satellite receiver, satellite-bone system is damaged.

Assuming that $\mathrm{P}_{\mathrm{e}}=1 \mathrm{GW}, \mathrm{G}_{\mathrm{e}}=40 \mathrm{~dB}, \mathrm{~B}_{\mathrm{e}}=500 \mathrm{MHz} . \mathrm{B}_{\mathrm{r}}=20 \mathrm{MHz}, \mathrm{G}_{\mathrm{r}}=30 \mathrm{~dB}$, and transmission loss is calculated from Formula (2). Assuming that EMP's transmitting antenna faces the antenna main lobe of targeted satellite receiving antenna directly and receiving area is $1 \mathrm{~m}^{2}$. The frequency of EMP is $5 \mathrm{GHz}, 5.75 \mathrm{GHz}$, and $6.5 \mathrm{GHz}$, respectively. The distance from satellites is $100 \mathrm{~m}$ to $1 \mathrm{~km}$. The above-mentioned parameters are substituted into Formula (1), the relationship between power density and acting distance coupling into the inner targeted satellites is shown in Figure 3:

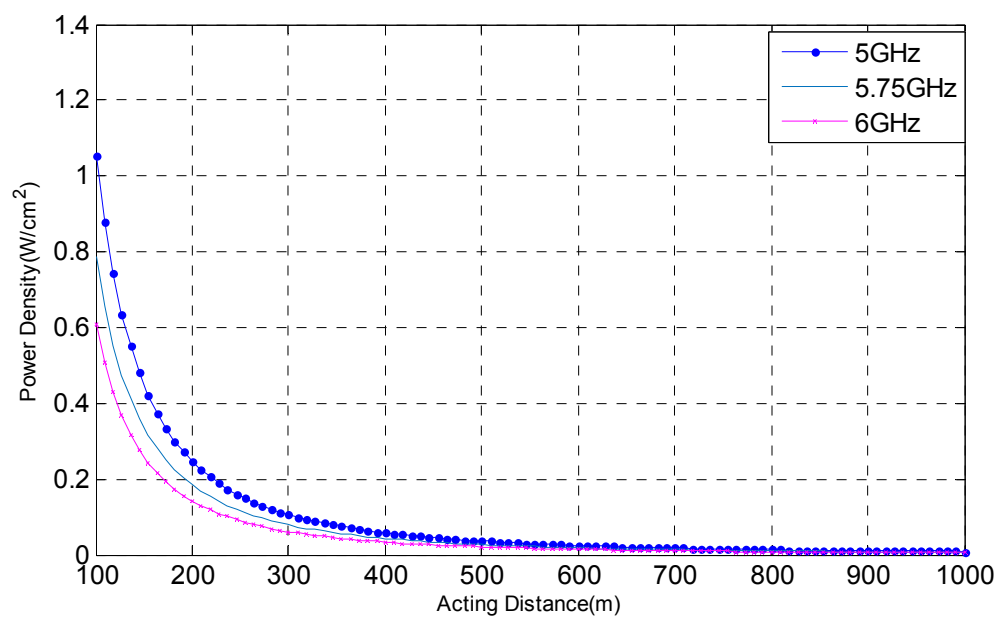

Figure 3: The Relationship among Working Efficiency, Acting Distance and Power Density

When frequency of EMP belongs to the bandwidth of satellite receiving antenna, the pulse enters into the effective load system of satellites through receiving antenna. Therefore, the first damaged one may be the receiver. The low noise amplifier with high frequency in receiver front end is the easiest damaged part. It can be observed from Table 1 and Figure 4 that when EMP coupling into the inner receiver from antenna, it may generate stronger disturbance on the receiver or burn it on the spot, resulting in abnormal work of effective load system for satellites.

\subsection{Star Sensor Coupling}

In order to study the basic principle of star sensor microwave coupling, the simulation model as shown in the figure is established. The thickness of metal shielding cavity is $t=2 \mathrm{~mm}$. The box size of inner size is $a^{*} b^{*} d=170 \mathrm{~mm} * 120 \mathrm{~mm} * 160 \mathrm{~mm}$. Here, $a, b, d$ are the length, width and height of rectangle shielding cavity. The focal length of star sensor is, lens hood size is, internal and external hole diameter of lens is $20 \mathrm{~mm}$ and $40 \mathrm{~mm}$. The stimulus wave parameter of EMP signal is: Trise $=1.67 \mathrm{e}-3$ (us), Tfall $=2.5 \mathrm{e}-2$ (us), Ttotal $=5$ (us), Amplitude $=6500$. 


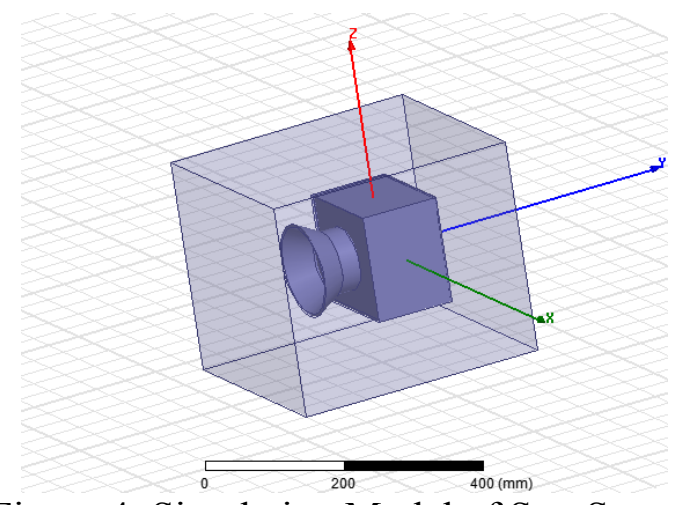

Figure 4: Simulation Model of Star Sensor

Stimulate to select plane electromagnetic wave to be incident. Its electric direction of polarization is parallel with the $\mathrm{z}$ axis direction. When giving no consideration to incident angle. Electromagnetic wave adopts front to be incident, namely incident vector $\mathrm{k}$ is vertical to the aperture vertically.

This model is conducted simulated within the frequency range of $10 \mathrm{GHz}$, the electric field strength and xoy plane electric field. This model is simulated within the frequency of range of $100 \mathrm{GHx}$, the field strength of electric field in star sensor cavity center is shown in Figure 5 and Figure 6, respectively. It can be observed from the figure that as frequency $4.2 \mathrm{GHz}$ and $4.8 \mathrm{GHz}$, electric field intensity of multiple frequencies preach the peak value, indicating that electric field intensity at the cavity center is greater than incident power strength and appears increased effect of electric field.

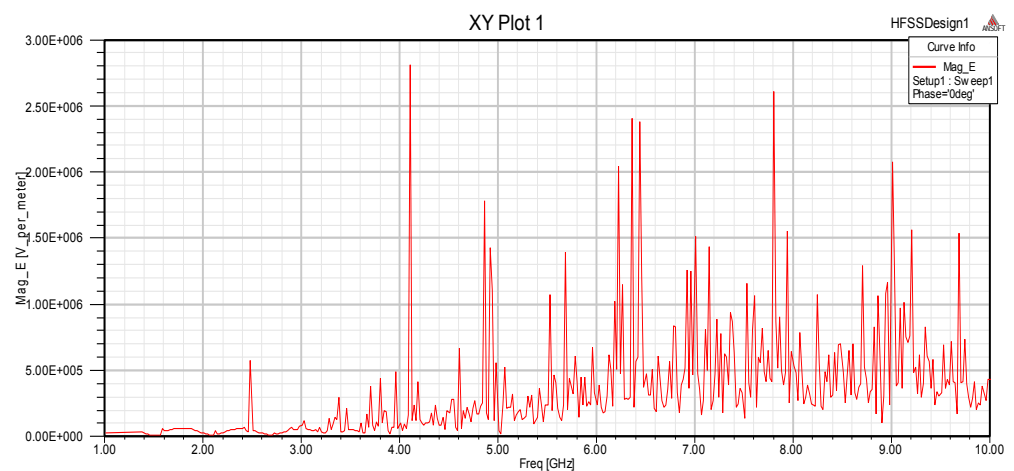

Figure 5: Electric Change Diagram in Cavity Center

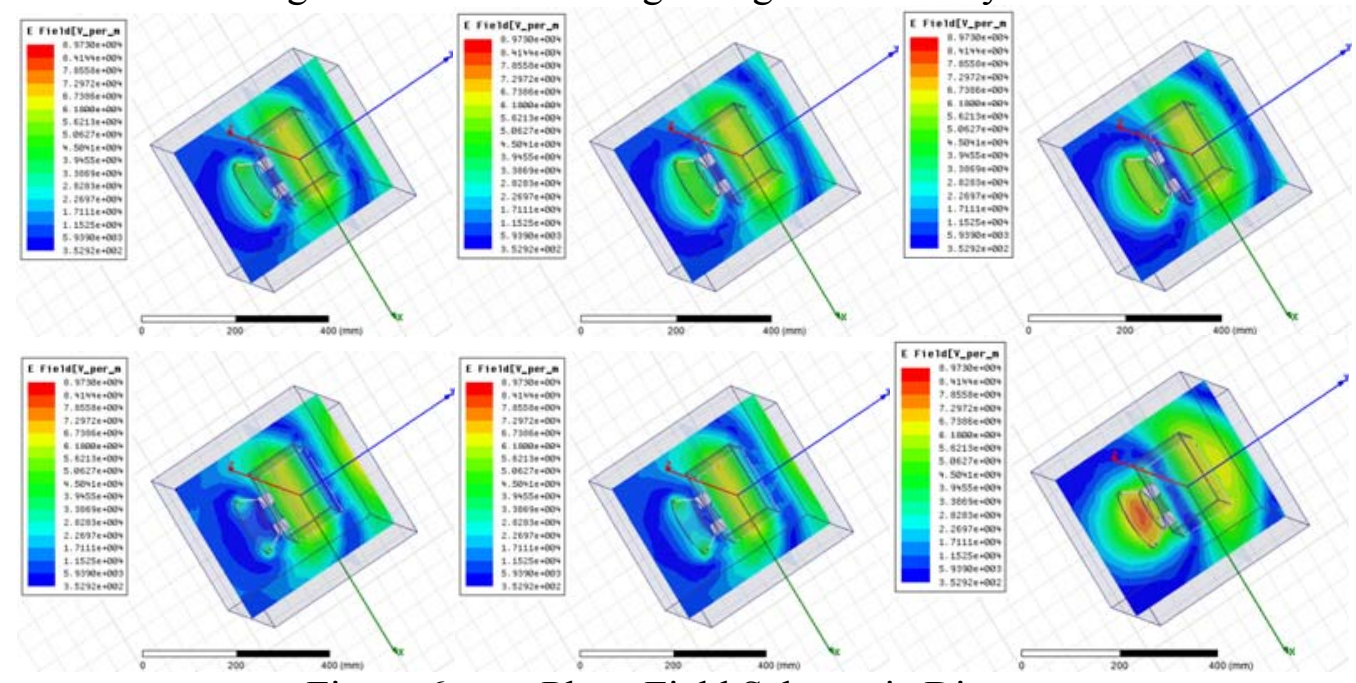

Figure 6: xoy Plane Field Schematic Diagram

The simulation results show that after EMP coupling into star sensor, it spreads to cavity back wall and forms vibration, then it radiates outward through a window, so that electromagnetic energy in the cavity is reduced gradually. When cavity center locates in the biggest power density, it is about $2800 \mathrm{kv} / \mathrm{m}^{2}$, in other words, $2.8 \mathrm{v} / \mathrm{cm}^{2}$, and it has stronger disturbance and damaging effect on the sensitive components of star sensor, especially it concentrates larger field concentrated on the 
top of element, it will cause larger disturbance and damage it.

\subsection{Transmission Line Coupling}

For cable coupling, it is based on the transmission line on the Sun sailboard. Assuming that length and width of sun sailboard is $600 \mathrm{~cm}$ and $240 \mathrm{~cm}$, respectively, the length of cable is $550 \mathrm{~cm}$. The transmission line model is shown in Figure SPICE model, respectively. EMP parameters are $65 \mathrm{kv} / \mathrm{m}$, Trise $=1.67 \mathrm{e}-3$ (us), Tfall $=2.5 \mathrm{e}-2$ (us), and Total $=5$ (us), amplitude $=65000$

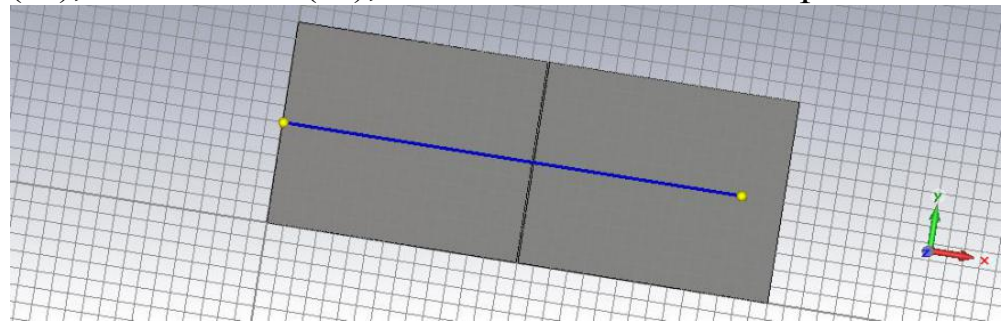

Figure 7: Transmission Line Mode for Sun Sailboard;

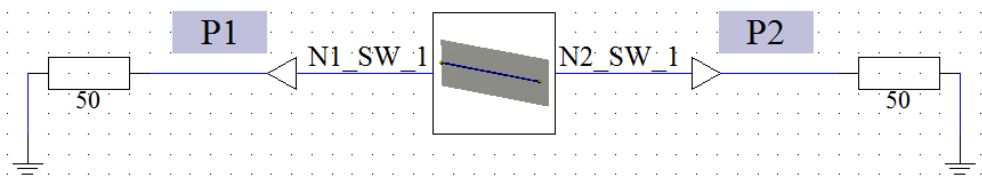

Figure 8: Cable Coupling SPICE Model

Through the transmission terminal load ya is shown in Figure 9
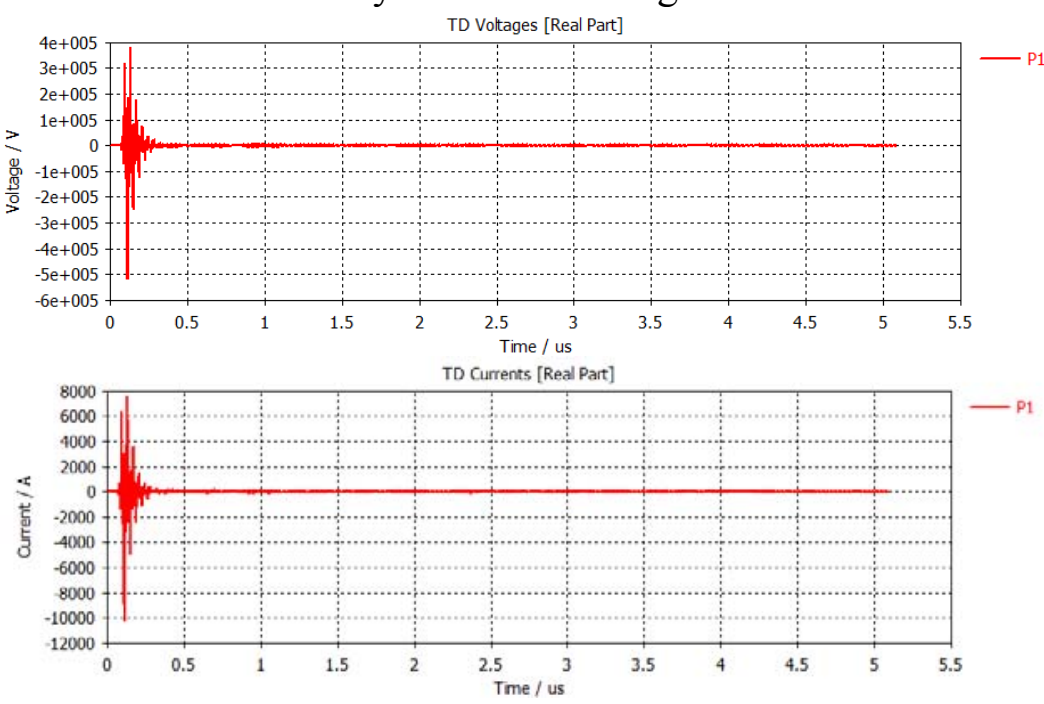

Figure 9 End Voltage and Current on the Terminal of Transmission Line

Through the simulation results, it can be observed that transmission line can generate voltage with the kv two grades and several thousand Ampere's current to act on the impedance of $50 \Omega$. The load peak power can reach several hundreds of watt. If electromagnetic energy coupling in the inner satellite system, it will cause a huge threat on electronic components.

\section{Summary}

It can be observed from the above-mentioned analysis and simulation calculation that there are multiple paths of coupling strong EMP and satellites. In the strong EMP, orbit satellites without electromagnetic reinforcement will be threatened seriously. The influences of EMP on satellites are the result of comprehensive effect. Therefore, it must consider survival of satellite system in strong electromagnetism, while establishing the satellite system. 


\section{References}

[1] Cao Lei, Analysis of Antenna's Property on EMP Response Based on FDTD[D], Harbin Engineering University, 2012;

[2] Yang Yuchuan, Threats and Protective Technique of High Power Microwave Weapons on Satellite Electronic System[D], National University of Defense Technology, 2008;

[3] Pang Yongjun, Prevention from "Pearl Harbor" Attack Event_American Satellites' Possible Measures on NEMP [J], Modern Military, 2005, 8:012

[4] Xu Fuxiang, Generality of Satellite Engineering[M], China Aerospace Press, 2003;

[5] Wu Gang, Song Zhigang and Liu Bo, Feasibility Analysis of High Power Microwave Weapons' Attacking Satellite Effective Load System[J], Modern Defence Technology, 2011, 39(5):16-20

[6] Pu Gehong, Jiao Yanping and Cheng Bo, Ability Evaluation on American Militaries' High Power Microwave Weapons to Impact Satellites' Weapon[J], Journal of Equipment Command Technology College, 2008,18(6):37-40;

[7] Zhao Guoqing, Radar Countermeasure Principles[M], Xidian University Press, 2001 